\title{
Interface Between Macro and Nano Worlds
}

\author{
doi:10.3991/ijoe.v6s1.1381 \\ G. Škorc ${ }^{1}$, R. Šafaric ${ }^{2}$, D. Lukman ${ }^{2}$, S. I. Protsenko ${ }^{3}$ and S. Zapušek ${ }^{2}$ \\ ${ }^{1}$ UPR d.o.o. \& Co. k.d., Slovenia \\ 2 University of Maribor/FERI, Maribor, Slovenia \\ ${ }^{3}$ Sumy State University, Sumy, Ukraine
}

\begin{abstract}
This paper describes the design of a nanorobotic application which includes: nanorobotic arm, Akiyama sensor system, a vacuum chamber, and an interface tools for the nanorobotic hardware developed using $\mathrm{C}++$ and VRML languages in order to create a desktop virtual-reality environment which improves visualisation and prevents collision of the nanomanipulator hardware with the associated workspace. The presented experiment shows how the used man-machine interface could be used for communication between macro and micro/nano worlds.
\end{abstract}

Index Terms-man-machine interface, nano robot, sensing nano objects.

\section{INTRODUCTION}

The micro/nanorobotic experiment is the very last in a sequence of telerobotic experiments, done in our laboratory. It is not a real telerobotics experiment, because the distance between the client's computer and a nanorobot is less than 1 metre. But the experiment could be treated as a telerobotics experiment because it connects two different worlds, the macro world of the user and the micro/nano world of the micro/nanorobot.

The focus of the presented micro/nanorobotic experiment is that the user cannot see the micro/nanorobotic object's movements etc [14], [15]. The $3 \mathrm{D}$ on-line image of the gripper and the object, even with powerful microscopic visual magnification, cannot be properly seen, due to the fact that the focused images of the objects and the nano-robotic tip tool can only be seen only within a narrow vicinity (few hundred $\mu \mathrm{m}$ ) of the microscope's optical lens focus. So, the same techniques [1], [2], [3], [4], [5], [6], as for the real telerobotics experiment were used in order to make a connection between the macro and micro/nano worlds. In fact, we used the telerobotics platform developed for the previously developed telerobotics experiment using a haptic device [5]. The reason, we used the haptic device is that we also like to include a sense of touch in the virtual teach pendant, in order to make communication between the human and the nanorobot easier and more userfriendly. Of course, the produced force from the haptic device is inadequate as a force produced during a collision in the micro/nano world, but it is magnified several times, so the human sense of touch can detect any collision within the micro/nano world [12], [16]. The hardware of the nanorobotic application is presented in Figure 1.

\section{DESCRIPTION OF THE NANOROBOT HARDWARE}

\section{A. Block scheme of the nanorobot predictive display}

The Figure 2 describes the block scheme of the micro/nanorobot operation system with predictive display. The human operator uses the blocks Model and Display as a visualization tool, where the virtual presentation of the real micro/nanorobot workcell with micro/nano objects can been seen. The block Model is also used a tool to prevent the collision of the micro/nanorobot arm (a gripper) with its environment. The block Sensors uses the Akiyamo probe for 3D measurement of the micro/nano object in the micro/nanorobot workspace. It is also used for calibration between real and virtual worlds (blocks Environment and Model). The block Master arm presents the haptic device, which is used for the user-friendly manmachine communication, so user also has the feeling of touch in the virtual world model. The block Slave arm is presented by our real lab micro/nano robotic arm.

\section{B. Micro/nanorobot cell}

The micro/nanorobotic system is divided into two parts (see Figure 3). The upper part of the figure consists of a real-time target application with a micro/nanorobotic cell: a development computer machine marked as 1, a real-time controller computer card marked as 2, and a target computer application marked as 3 .

The usual notebook computer marked as 1 is used as the development computer for control algorithms and user interfaces with an installed Windows XP operating system and a LabView 8.5 software package.

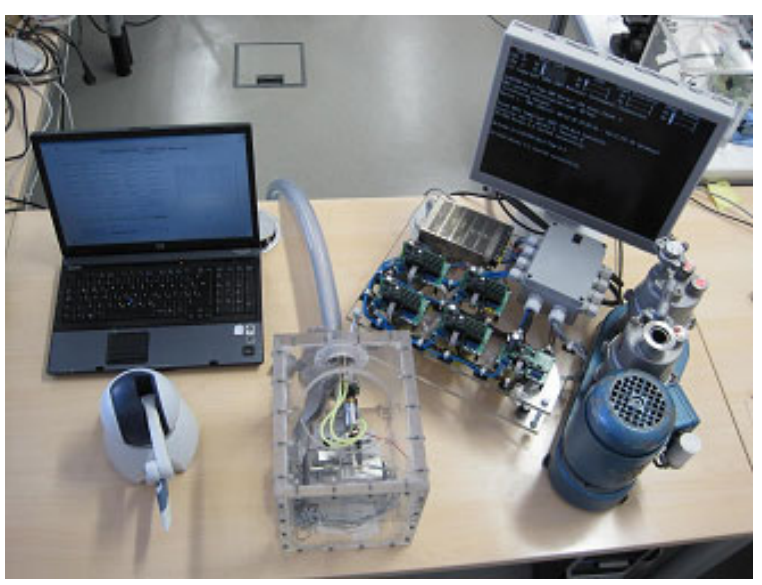

Figure 1. The whole micro/nanorobotic application 
Optical picture magnification

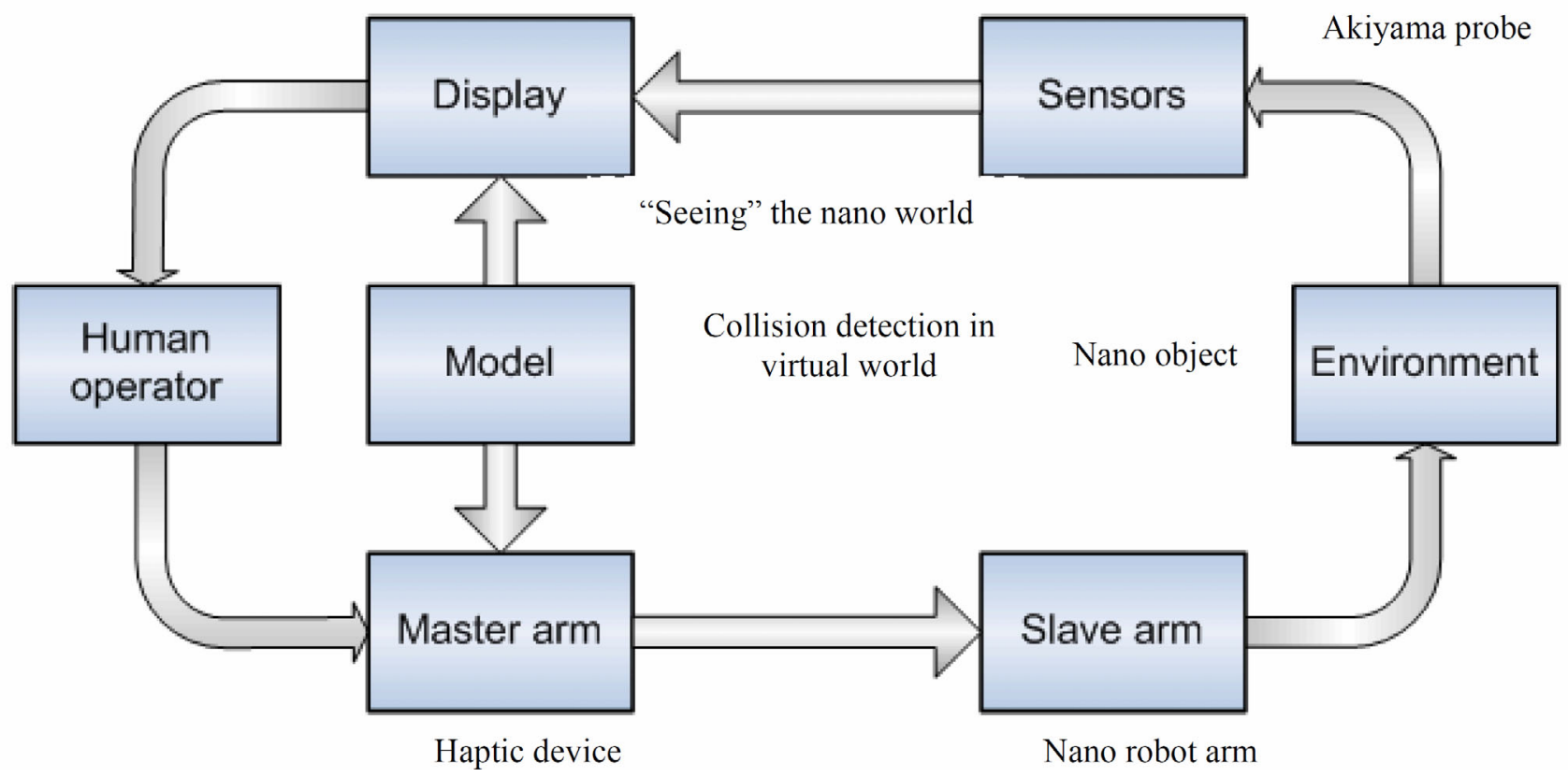

Figure 2. Block scheme of the nanorobot operation system with predictive display

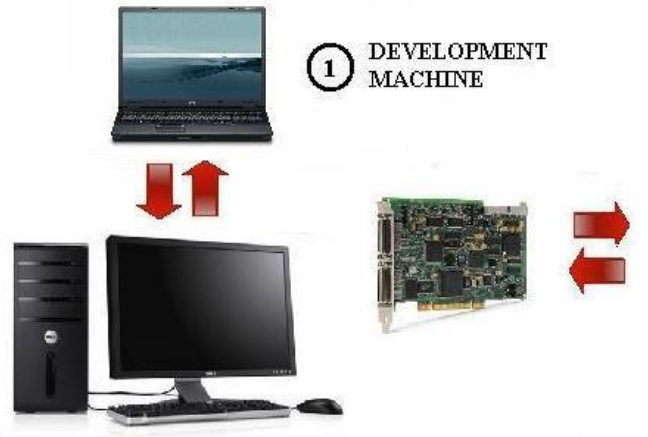

(2) REAL TIME TARGET PC + PCI7356

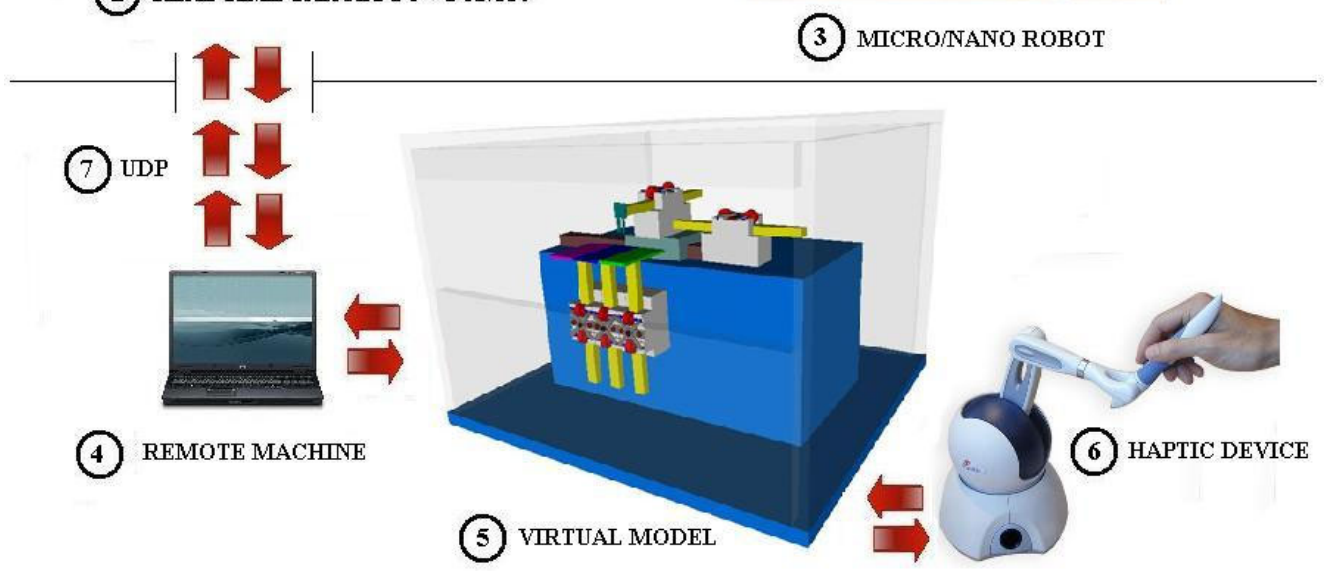

Figure 3. Micro/nanorobot system components 
The PC computer, marked as 2, executes real time control algorithms using so-called Real Time Desktop Target software. This PC runs a LabView Real Time operating system, which is independent of all the other operating systems already installed on the PC. Our realtime target PC is supported by a 7356 PCI motioncontroller card from the National Instruments Company.

This card is used as an interface between the control algorithm and the piezoelectric motor-power drivers. Its output has a separate reference signal for piezoelectric motor drive in regard to each axis of the controlled system for all 5 axes and is calculated by signals from the position of feedback and desired position inputs using the control algorithm.

The execution time of the control algorithm is about 3 $\mu \mathrm{s}$ [7]. The micro/nanorobotic cell, presented as 3 (Figure 3 ), is actuated by five linear piezo-motors produced by the PiezoMotor Upsala AB Company, where the motor movement steps can have lengths from $2 \mathrm{~nm}$ to $8 \mu \mathrm{m}$ and can achieve speeds of up to $12.5 \mathrm{~mm} / \mathrm{s}$ using factorydelivered demo-drive electronics [8]. The construction of the nanorobotic cell is done in such a way that two of the piezo-motors act as $\mathrm{X} / \mathrm{Y}$ manipulators, and the other three are used as motion drivers for three separate serving tables within the $\mathrm{Z}$ axis. Positional feedback values for all axes are achieved using electro-magnetic linear encoders produced by the NANOS Instruments Company. Each encoder-set's electronics are built using magnetic scale and sensor electronics. The linear position instrument has a resolution of $61 \mathrm{~nm}$ with precision of $+/-0.15 \%$ [9].

The micro/nanorobotic cell allows implementation of different micro and nanorobotic tip tools. A special two finger gripper is used temporarily for gripping objects of micro-scale sizes $(100 \mu \mathrm{m}$ to $200 \mu \mathrm{m})$. It is placed at the top of the $\mathrm{Y}$ axis [10].

\section{Vacuum chamber}

For purposes of testing some experiments (Akiyamo probe sensing, transportation of the nano objects) in a vacuum, we have build a special low cost vacuum chamber. It was designed to withstand the middle vacuum of approximately 6 mbar. It is built out of a $15 \mathrm{~mm}$ thick plexi glass with polished edges sealed with silicon kit. It has an openning of $135 \mathrm{~mm}$ diameter with sealed aluminium top, which allows the entry of the micro/nano robotic cell.

Vacuum chamber alone can withstand the pressures up to 1,9 mbar. However for purposes of our nano robotic cell application, we have to add some other features. First are the wire connections for a robotic cell, which is made out of 5 piezo motors and 5 electromagnetic encoders. We also have to seal cable for video camera and a couple of tubes for cooling of some components. This results in more than 60 wires and tubes to seal. There is of course the possibility to buy such feedthroughs on the market, but they are built for high vacuum and have of course correspondingly high price. While we need only middle vacuum of 6 mbar, we tried to make the low cost version of electrical feedthroughs.

The problem at sealing wires at such vacuum is that one can seal the outer insulation of the wire, but not the internal bundle of thin wires. This is why it is necesary to seal only one thick wire (see Figure 4).

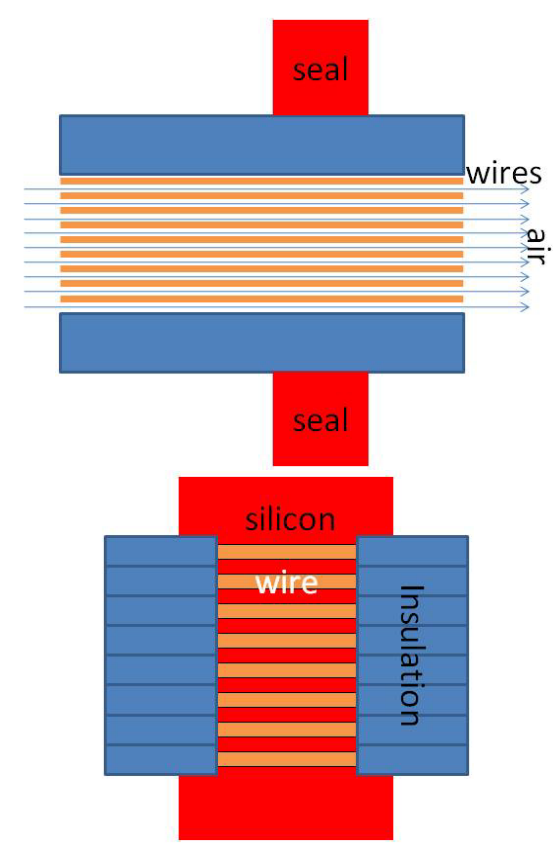

Figure 4. Constructing the sealed wire connections

The brass tubes were also sealed by a silicon kit. On such way it was possible to achieve about 4,5 mbar of total pressure in the chamber. We assume that some wires were not sealed perfectly and therefore comes to a higher pressure in the chamber. We assume that it has to be possible to achieve initial 1,9 mbar with or without feedthroghs. At all experiment rotary vane pump has been used. The vacuum chamber can be seen in Figure 7 (B,C and D).

\section{3D Akiyama scanner for virtual modeling of micro/nano parts}

Scanning probe microscopy (SPM) is one of the today's well known techniques for acquiring images with submicrometer or even sub-nanometer resolution. Atomic force microscope (AFM) is one of the oldest and probably most known microscopes from SPM techniques. It uses very sharp probe (with tip diameters of up to one atom), which deflects when it is brought near the measured sample surface. Deflection of the probe, which is caused due to presence of different forces (van der Walls forces, capillary forces, electrostatic forces, etc.) between the tip of the probe and sample surface, is measured with an optical laser system in continuation. Sophisticated AFM optical measuring system and probe exciting system has led to a development of a novel Akiyama probe [17, 18]. This probe is based on a self sensing and actuating quartz tuning fork, combined whit a micro-machined cantilever (Figure 5).

It is used for a dynamic mode AFM, where height of the sample is measured according to frequency shift from the basic resonance frequency.

Because probe combines self actuating and self sensing principle it can be easily integrated into different industrial systems [19]. With $310 \mu \mathrm{m}$ of length, $28 \mu \mathrm{m}$ of height and $15 \mathrm{~nm}$ of tip diameter it occupies very small space above the sample and allows different approach principles to the sample [20], [22], [23]. All these good characteristics of the probe have inspired us, to start a development of a so 


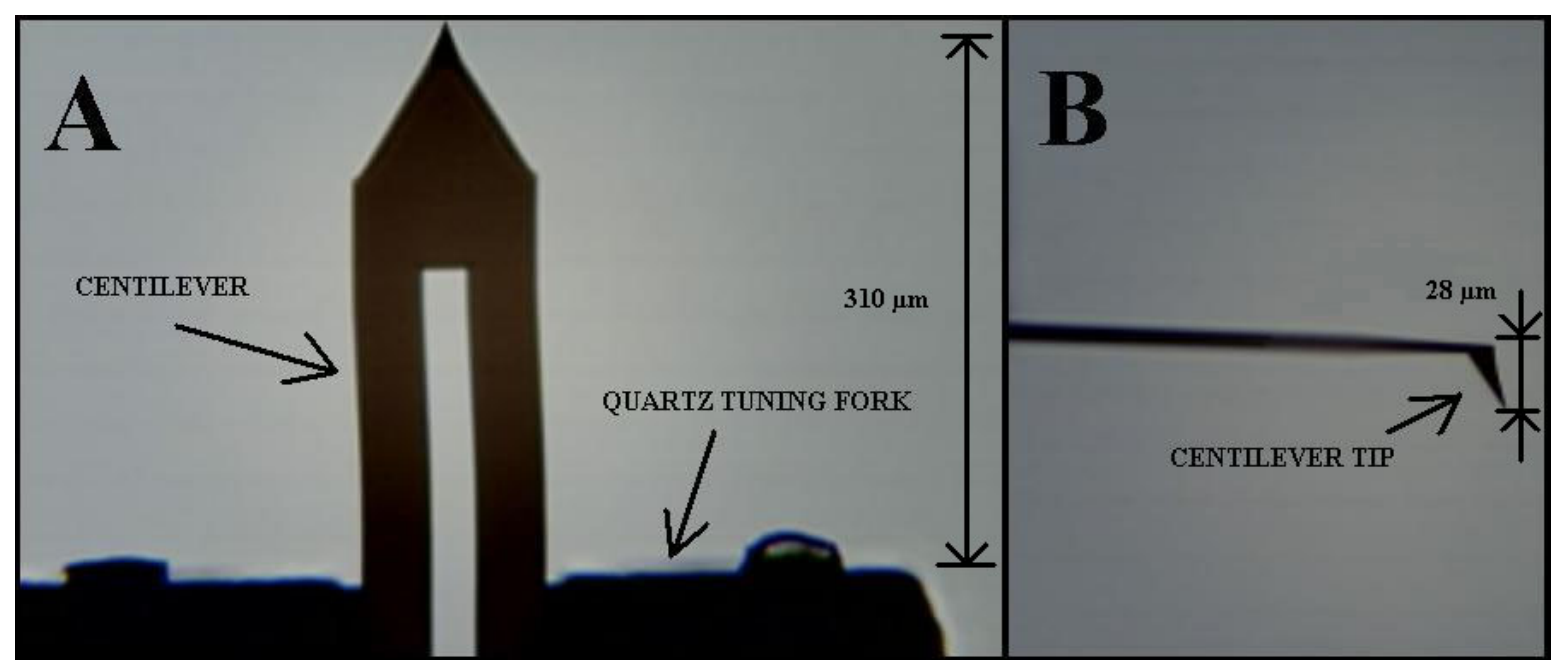

Figure 5. Top (A) and side (B) view of the Akiyama probe

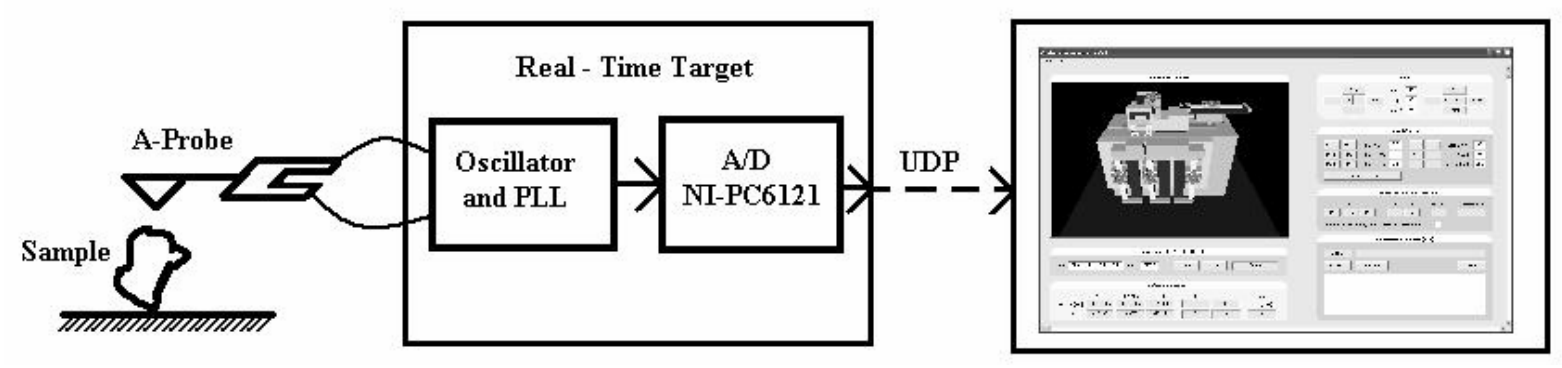

Figure 6. Concept of 3D Akiyama scanner for virtual modeling of micro parts

called 3D scanner for virtual modeling of micro/nano parts.

Basic idea of this new application is an upgrade of existing application presented in section B (Figure 6). Upgrade has been made in two steps. First Hardware step includes implementation of self oscillation and PLL circuit built according to schematic presented in [20]. Analog signal received from PLL circuit is acquired with NI-PCI6121 A/D card [21] which is integrated into real time target PC presented in section B. In second upgrade step, two software upgrades is needed. First one is made at real time target, where another algorithm for acquiring and scaling of analog signal from Akiyama probe is added. Digitalized information about sample height is sent using UDP to the remote application. The second software upgrade is made at user interface presented in section III. Here we had to rebuild user interface on that way that it would be able to read digitalized information received from the target application. This information is then transformed into VRML model and shown in our user interface in continuation. So developed application will allow us implementation of new functions and safer experimenting with objects of small sizes (in virtual space no damages can be made to micro/nanorobotic manipulator or micro/nano objects).

Actual situation of our application is presented on Figure 7. It is divided in 4 sections, where section A presents the robotic controller, section $B$ the top view of vacuum chamber, section $C$ the robotic manipulator inside of the vacuum chamber and section $\mathrm{D}$ the side view of the robotic application.

\section{MACRO - NANO WORLDS INTERFACE}

The second part of the application, as presented in the lower part of Figure 3, shows a man-machine interface. It consists of a "remote computer" marked as 4 , where a virtual model of the micro/nanorobotic cell, marked as 5 and a haptic-device, marked as 6 , have been installed. The "remote computer" is a notebook, based on the Windows XP operating system, supported by a Microsoft Visual $\mathrm{C}++$ software package and OpenHaptics software package from the Sensable Technologies Company. Both packages with the PC hardware are used for developing and executing the micro/nanorobot application. A UDP protocol, marked as 7 , is used to ensure the fastest and reliable enough communication between the user and the machine. A virtual VRML model of the micro/nanorobotic cell, marked as 5 , is also included in the application, because the user does not see the target application - manipulation of the micro sized objects on all three serving tables with the naked eyes. The user doesn't have a real feeling of what is exactly happening within the target application.

The virtual model gives a user 3D magnification and visualisation of the objects, including a robotic tip-tool within the target application. The virtual model of the micro/nanorobotic cell with its static macro/nanorobotic environment is updated with the on-line positional data acquired from the real micro/nanorobotic cell application. So, the user can visualise a $3 \mathrm{D}$ on-line image of the object's current positional situation and macro/nanorobotic tip-tool movements from different viewing points. This is needed, because otherwise we 


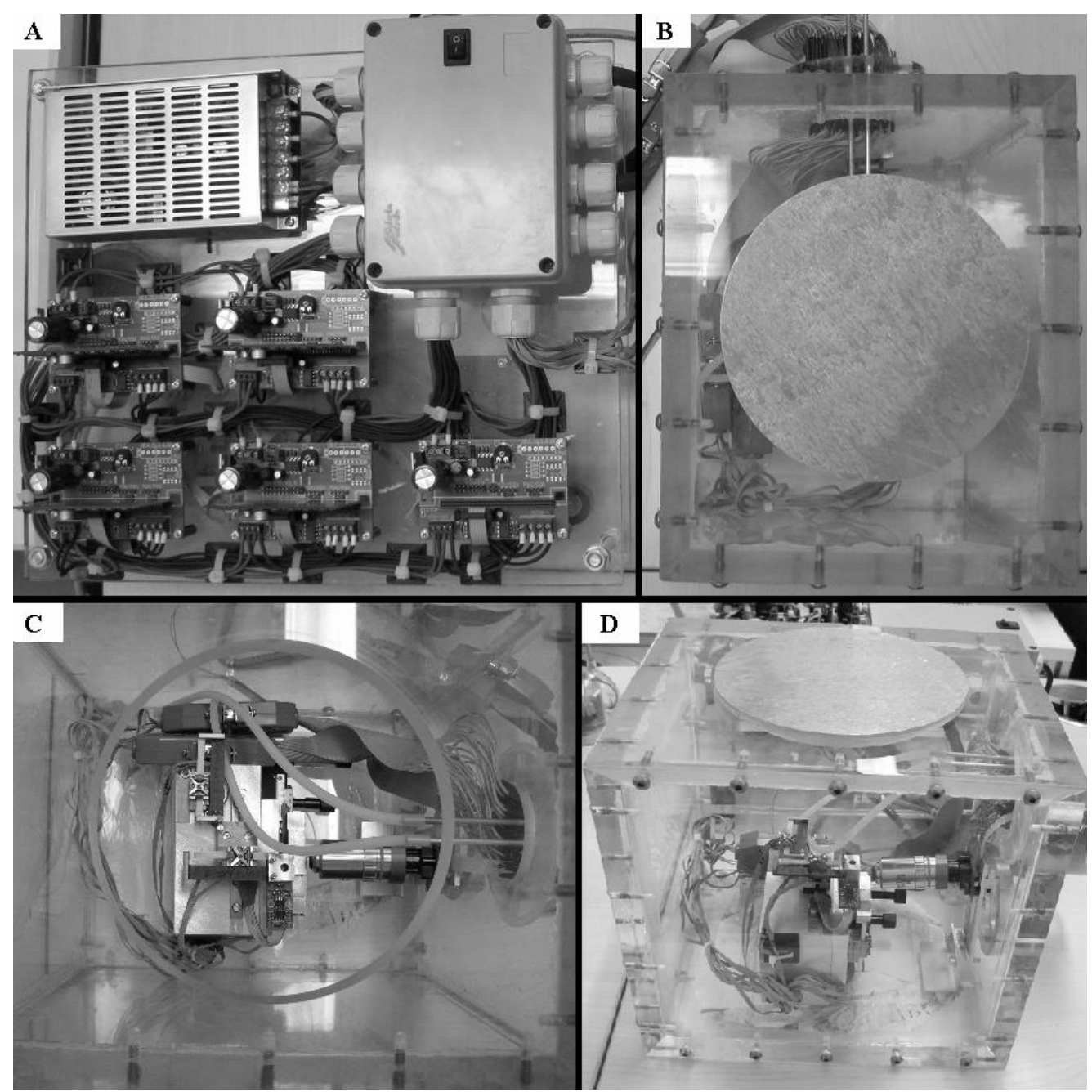

Figure 7. An actual situation of our micro/nano robotic application

would need to use a microscope which could provide focused images of the objects and the micro/nanorobotictip only within a narrow vicinity (less than $500 \mu \mathrm{m}$ if we use an optical magnification factor between 10 and 25) of the microscope's optical lens focus. The micro/nanorobot's workspace is a little less than $3 \mathrm{~cm}^{3}$. The second reason for using a virtual model of the micro/nano robotic cell is for an off-line programming of it, which gives the user the possibility studying the target application program, to improve it, to test it and, after that, to send it for execution in the real-world.

The micro/nano robotic cell system uses a Phantom Omni haptic device in order to provide the user with a more realistic feeling about the situation inside the micro/nano-robot workspace. The user can control the haptic device by moving its arm along 6 degrees of freedom (X, Y, Z, roll, pitch and yaw). Three motors are built onto the $X, Y$ and $Z$ axes to give the user forcefeedback information as a virtual presentation of friction or space limits or collision between micro/nanorobot axes or between the micro/nanorobot tip tool with the object in the virtual world. The chassis of the haptic device has two free programmable built-in buttons which are used to grab, move, create different viewing points etc, of a single axis of the micro/nanorobotic cell's virtual model.

Figure 8 takes a close look of the current real micro/nano robotic tip gripper. It is made of so called structural glass [10], [11], driven by a piezoelectric motor, which is situated horizontally to the vertical fingers of the gripper. The piezoelectric actuator allows the fingers to be moved approximately $100 \mu \mathrm{m}$. So, the gripping distance between fingers is between $100 \mu \mathrm{m}$ and $200 \mu \mathrm{m}$. It was specially designed to grip and move optic fibre glasscables with diameters between $100 \mu \mathrm{m}$ and $125 \mu \mathrm{m}$.

Figure 9 presents a screenshot of the so-called Control teach pendant or man-machine interface. This interface is divided into 7 sections:

- The implemented VRML model of the micro/nanorobotic cell can be seen in the upper left corner of Figure 9.

- The viewing section (right upper corner of Figure 9) is used for selecting the most appropriate viewing point for the VRML model of the micro/nanorobotic cell. Integrated virtual buttons allow the user to translate or rotate the view. A zoom function is also available within this section. Manual control section (beneath the viewing section) is used for moving each axis using two virtual buttons. The first one is used for moving the axis one step forward and the other for moving one step backwards. The lengths of motor steps can be set individually by typing the length number in millimetres in the small windows near the virtual buttons. 


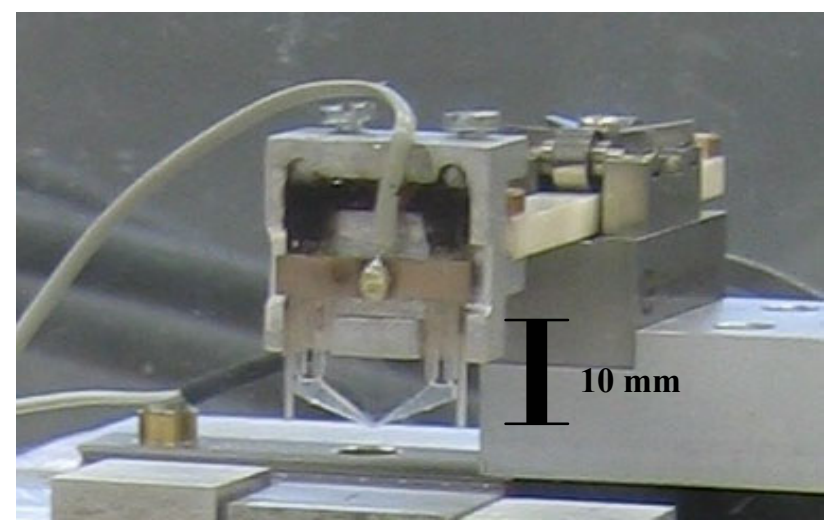

Figure 8. Close look of a real micro/nano-robot tip gripper

Control of the haptic-device section (beneath the manual control section) is used for selecting the axis, which will be controlled using the haptic-device. This function is also available on the haptic-device by using one of the buttons integrated on the haptic-device's chassis. The number of the selected axis is shown within this section.

PTP regime control section (lower right corner of Figure 9) allows the user to load a file within the given micro/nano robotic cell program using PTP trajectory coordinates. Movements are executed sequentially, step by step, as they are programmed and written in the file. Any positional changes of any axis within the described control sections are animated in the virtual model of the micro/nanorobotic cell.

On-line/off-line section gives the user the possibility of simulating programmed micro/nanorobotic cell target applications before they are sent to the real micro/nanorobotic cell. Of course, this option is only provided if the off-line virtual button is selected. Here, any changes within any control-section are executed as a simulation of the micro/nanorobotic cell's virtual model. When the user enacts the on-line regime, the given changes are executed on the real machine, immediately. This off-line function is very helpful when the user programs and tests certain new trajectories in the micro/nanorobotic cell's workspace, and when it is unsure what consequences some newly-programmed movements would have (collision inside workcell space, movements outside workcell space etc).

The last section is the reference position section, where the user can set axis reference positions in certain specific cases, where the default given references are unacceptable.

Another user interface, the so-called Monitoring teach pendant (see Figure 10) is built on the same basis as previously described The control teach pendant can only provide a function for axes' actual positional monitoring on an already executed target application. It also has a virtual model of the micro/nanorobotic cell, so the user can visualise the real micro/nanorobotic axes, and tool movements. This interface uses a $\mathrm{C}++$ built receiver to animate an implemented VRML model. The $\mathrm{C}++$ receiver receives the actual positions of all five axes and the gripper from the real micro/nanorobotic cell, during the programmed target application execution, and delivers these positions to the VRML model, where they are animated. A viewing section is also available in the Monitoring teach pendant, where user can choose different angles for viewing and zooming. All other functions, which are available within Control teach pendant are disabled here.

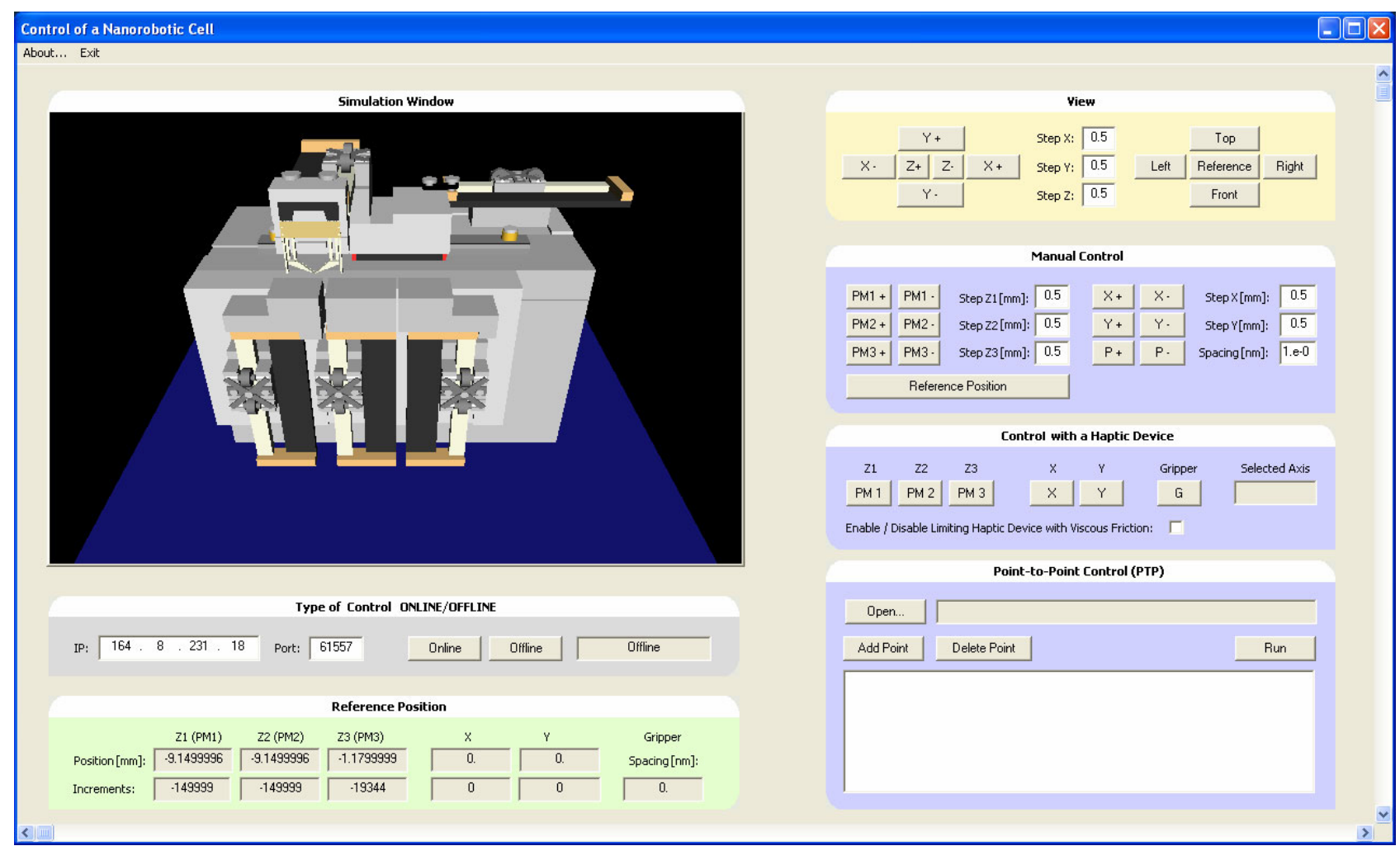

Figure 9. The Control teach-pendant of the nano-robotic application 


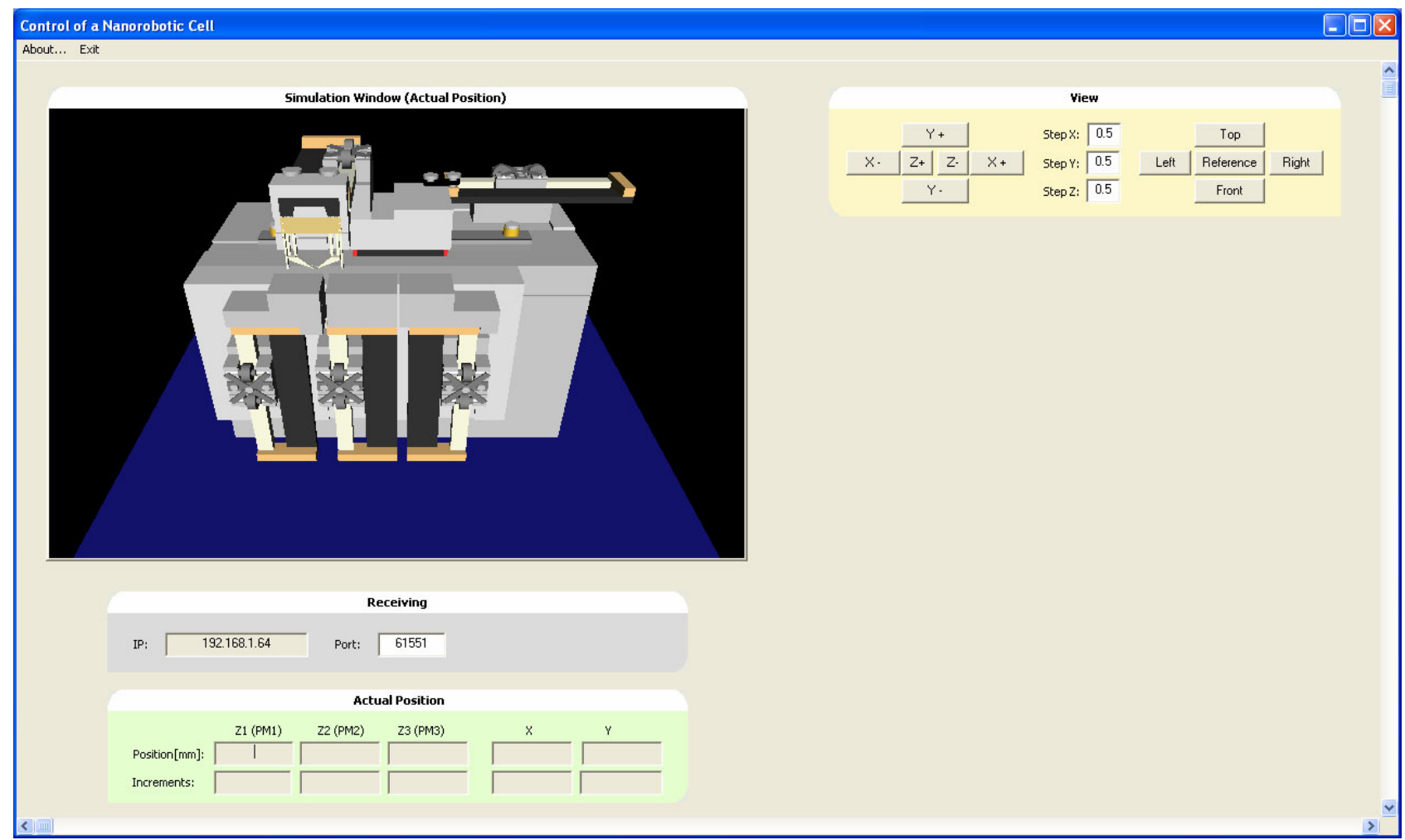

Figure 10. The Monitoring teach-pendant of the micro/nanorobotic application

\section{CONCLUSIONS}

The paper has presented the development of a nanorobotic application using an implemented VRML virtual model. This virtual model is used for the visualisation of real-time actual or desired remote robot positional control data, and is used as a replacement for live camera pictures. The human machine interface for connecting of macro and micro/nano worlds was presented and where a virtual model of the micro/nano robotic cell is used to improve $3 \mathrm{D}$ visualisation. The use of a haptic device remarkably improved the communication link between the user and the micro/nanorobot.

The main drawback of the presented method is that they can prevent collision, or provides feeling of touch, only within predefined robotic cell and its environment, while it is very difficult or even impossible to sense or visualize so-called unknown dynamic objects.

\section{REFERENCES}

[1] J. Čas, D. Hercog, R. Šafarič, "Web based education tool for neural network robot control," Int. j. online engineering., Vol 3, no. 2, 8 f, 2007, (http://www.i-joe.org/ojs/).

[2] R. Bambang, "Development of Architectures for Internet Telerobotics Systems," ICIUS 2007, pp. 137-142, 2007, (http://arxiv.org/ftp/arxiv/papers/0804/0804.3891.pdf)

[3] R. Šafarič, D. W. Calkin, R. M. Parkin, C. A. Czarnecki, "Virtual environment for telerobotics," Integrated. computer-aided engineerin, Vol. 8, pp. 95-104, 2001.

[4] R. Šafarič, M. Debevc, R.M. Parkin, S. Uran, "Telerobotics experiments via Internet," IEEE transactions on industrial electronics, Vol. 48, pp. 424-431, 2001. doi:10.1109/41.915422

[5] R. Šafarič, S. Šinjur, B. Žalik, R.M. Parkin, "Control of Robot Arm with Virtual Environment via Internet," Proc. I.E.E.E., pp: 422-429, 2003.
[6] R. Šafarič R, M. Truntič, D. Hercog, G. Pačnik, "Control and robotics remote laboratory for engineering education," Int. $j$. online engineering, Vol. 1, 2005, (http://www.i-joe.org/ojs/).

[7] National Instruments, Motion controller 7356 datasheet web page: http://www.ni.com/pdf/products/us/735x.pdf

[8] Piezomotor Upsala AB: PiezoLEGS data and user instructions, 3rd edition, pp. 3- 15, 2003.

[9] Nanos instruments web page: http://www.nanos-instruments.de/

[10] R. Keoschkerjan, H. Wurmus, "A novel microgripper with parallel movement of gripping arms," Actuator 2002, 8th International Conference on New Actuators, Bremen, Germany, pp. 321-324, 2002.

[11] G. Škorc, J. Čas, S. Brezovnik and R. Šafarič, "Position control with parameter adaptation for a nano-robotic cell," accepted, but still in the process by Journal of Mechanical Engineering

[12] Dong-Soo Kwon, Seung-Chan Kim, " Haptic Interfaces for Mobile Devices: A Survey of the State of the Art," Recent Patents on Computer Science 2008, pp. 84-92, 2008, (http://robot.kaist.ac.kr/paper/doc/b2670_gny6c48k_PDF.pdf).

[13] Seung-Chan Kim and Dong-Soo Kwon, "Haptic and Sound Grid for Enhanced Positioning in a 3-D Virtual Environment," I. Oakley and S. Brewster (Eds.): HAID 2007, LNCS 4813, Springer-Verlag Berlin Heidelberg 2007, pp. 98-109, 2007, (http://www.springerlink.com/content/5r125822426634lv/fulltext. pdf).

[14] T. Fukuda, T. Arai, "Prototyping Design and Automation of Micro/Nano Manipulation System," Proc. of IEEE Int'l Conf. on Robotics and Automation (ICRA'00), pp. 192-197, 2000.

[15] A, Cavalcanti, R. A. Freitas Jr., L. C. Kretly:, "Nanorobotics control design: A Practical Approach Tutorial,” DETC2004, pp. $1-10,2004$.

[16] (http://www.nanorobotdesign.com/papers/nanorobotTutorial.pdf).

[17] A. Cavalcanti, W. W. Wood, L. C. Kretly, B. Shirinzadeh, "Computational Nanomechatronics: A Pathway for Control and Manufacturing Nanorobots, “ CIMCA-IAWTIC'06, pp.185 - 190, 2006.

[18] T. Akiyama, U. Staufer, N. F. de Rooij, Self-sensing and selfactuating probe based on quartz tuning fork combined with microfabricated cantilever for dynamic mode atomic force microscopy, Applied Surface Science, Volume 210, Issues 1-2, 
5th International Conference on non-contact AFM in Montreal, Canada, 31 March 2003, Pages 18-21,

[19] Dara Bayat, Terunobu Akiyama, Nicolaas F. de Rooij, Urs Staufer, Dynamic behavior of the tuning fork AFM probe, Microelectronic Engineering, Volume 85, Issues 5-6, Proceedings of the Micro- and Nano-Engineering 2007 Conference - MNE 2007, May-June 2008, Pages 1018-1021, ISSN 0167-9317,

[20] Stucklin, S.; Gullo, M.R.; Akiyama, T.; Scheidiger, M.; "Atomic force microscopy for industry with the Akiyama-Probe sensor", International Conference on Nanoscience and Nanotechnology ICONN 2008, pp.79-82, 25-29 Feb. 2008.

[21] Nanosensor, Akiyama-probe (A-probe) guide, Version 2009-0323, retrieved from www.nanosensors.com on 12.01.2010,

[22] National Instruments, Low-Cost M Series Multifunction Data Acquisition - 16-Bit, $250 \mathrm{kS} / \mathrm{s}$, up to 80 Analog Inputs - datasheet, retrieved from www.ni.com on 28.06.2010.

[23] V. Matko, R. Šafarič, "Major improvements of quartz crystal pulling sensitivity and linearity using series reactance," Sensors, vol. 9, iss. 10, p.p. 8263-8270, 2009. doi:10.3390/s91008263

[24] V. Matko, K. Jezernik, "Greatly improved small inductance measurement using quartz crystal parasitic capacitance compensation," Sensors, vol. 4, iss. 10, p.p. 3954-3960, 2010. doi: $10.3390 / \mathrm{s} 100403954$

\section{AUTHORS}

G. Škorc is with the UPR d.o.o. \& Co. k.d., Krška cesta 8, 8311 Kostanjevica na Krki (e-mail: Gregor.Skorc@resistec.si).

R. Šafarič is with the University of Maribor, FERI, Smetanova 17, 2000 Maribor, Slovenia (e-mail: riko.safaric@uni-mb.si).

D. Lukman is with the University of Maribor, FERI, Smetanova 17, 2000 Maribor, Slovenia (e-mail: david.lukman@uni-mb.si).

S. I. Protsenko is with the Sumy State University, Sumy, Ukraine, (e-mail: protsenko@aph.sumdu.edu.ua).

S. Zapušek is with the University of Maribor, FERI, Smetanova 17, 2000 Maribor, Slovenia (e-mail: simon.zapusek@uni-mb.si).

This work was supported in part by Slovenian Government, TIA and ARSS agencies. Operation is partly financed by the European Union, European Social Fund.

This article was modified from a presentation at the REV2010 Conference at KTH, Stockholm, Sweden in June 2010. Submitted July 15th, 2010. Published as resubmitted by the authors July 29th, 2010. 